\title{
Integumentary System Signs and Symptoms
}

National Cancer Institute

\section{Source}

National Cancer Institute. Integumentary System Signs and Symptoms. NCI Thesaurus. Code C5036.

Patient complaints (symptoms), physical and/or laboratory findings related to the skin or integument. 